\title{
The Spleen: to Spleen or not to Spleen in Hematologic Diseases and in Vector Borne Hemoparasites
}

\section{Roy D. Meléndez}

Unidad de Investigación en Enfermedades Parasitarias (UNIPARVET).Decanato de Ciencias Veterinarias (DCV). Universidad Centroccidental Lisandro Alvarado (UCLA), Barquisimeto, Estado Lara. Venezuela

Key words: Splenectomy, Hemolytic diseases, Hemoparasites, Pathogenicity.

\section{Introduction:}

The spleen is an organ of the lymphatic system located in the left upper quadrant of the abdominal cavity of all mammals, above the stomach, and under the 9th and 11th left side ribs (Netter, 2011). The spleen has some important hematopoietic and immunological functions, since it produces red blood cells (RBC), destroys damaged and old cells, it recycles iron, and it filters blood. Microscopical studies showed that the splenic tissue, stained with Hematoxylin-Eosin (H\&E) has three defined areas: a) a thick external capsule, b) a reddish area, the "red pulp", where it occurs filtration of circulating RBC (Abbas et al., 2015); in addition, in mice there is a reserve of monocyte cells in this area (Swirski et al., 2009) and c) the "white pulp", an active immune response zone organized in lymphoid follicles rich in B- lymphocytes, together with large accumulation of $\mathrm{T}$ - lymphocytes around the periarteriolarsheats (Abbas et al., 2015). The red pulp of the mouse's spleen contains over the $50 \%$ of the body monocytes, whereas the white pulp synthesizes

\section{Abstract}

The objective of this work is to cogitate about the role of the spleen as a lymphatic organ which mainly acts in two ways: 1) as a natural filter in the circulatory system, 2) as an important protective organ of the immune system, destroying invasive bacteria, hemoparasites, viruses, and old and damage red blood cells (RBCs). Splenectomy (SPLY), the surgical extraction of the spleen, is often practiced to human patients who suffer different kinds of traumas or accidents. For many years the spleen was considered as a secondary lymphatic organ, which could be surgically removed from the abdominal cavity of traumatized patients causing low or none risk to their lives. This medical view has changed in the last 20 years as long as more scientific advances have occurred about the immunological, hematological, and protective functions carried out by the spleen as an essential and defensive organ in the circulatory system. Nowadays, a significant amount of SPLYs is practiced partially, unless a previous medical diagnosis decides that the extraction must be complete to preserve the patient's life. As per a recent study, splenic cells is found to control even the reproductive rate of an external parasite like the tick Amblyomma cajennense after infecting SPLY rats.

immunoglobulins, and it removes antibody-coated bacteria (Swirski et al., 2009). In some way the spleen can be considered as a giant lymph node, and the reactive center of activity of the Mononuclear Phagocyte System (MPS) (Delves et al., 2014).

\section{Embryonic origin of the spleen:}

The embryonic development of the spleen is from a condensation of the mesodermal mesenchyme, of the same lateral mesodermal plate that gives origin to the heart, limbs, lymph, blood, and adipose tissues (Patterson et al., 2000). The homeobox gene $\mathrm{NKx2}-5$ acts as a marker for spleen precursor tissues, thus pre splenic tissues are initially located in symmetrical domains on both sides of the embryo, and later, only the left side goes on to form the mature spleen.

A pool of progenitor cells is found in the spleen that derived from hematopoietic stem cells (HSCs), and allows a continuous production of blood cells. These cells are generated once in the lifetime from mesoderm derivatives 
closely related to endothelial cells, during a short period of embryonic development (Golub \& Cumano, 2013; Zhao et al., 2010). Besides, differentiation of HSCs first occurs in the fetal liver, giving rise to mature blood cells. HSCs also expand in the fetal liver, and in a short time period (four days in the mouse embryo), they increase over 40 -fold. HSCs and progenitor cells exit the fetal liver and colonize the spleen, where differentiation to the myeloid lineage and particular lymphoid subsets is favored (Golub \& Cumano, 2013; Zhao et al., 2010).

\section{Anatomy facts:}

the spleen macroscopically has a dark red to blue dark color, it has a triangular or a tongue-like shape in rodents, and the ratio of spleen weight to body weight is in general constant regardless of age, i.e. in rats is around $0.2 \%$ (Cesta, 2006; Moore, 2013), whereas the mean weight of the spleen in adult humans is $139 \mathrm{~g}$ (Moore, 2013; Molina \& DiMaio, 2012). The spleen is a highly vascular organ since it is a blood filter; blood enters the spleen at the hilus via the splenic artery, which divides into trabecular arteries located within the trabeculae entering the splenic parenchyma. Small arterioles branch from the trabecular arteries and enter the red pulp where they become central arterioles surrounded by lymphoid tissue. Smaller arterioles branch from the central arterioles and feed the white pulp capillary beds (Satodate et al., 1986).

Various pigments may be present in the spleen. Hemosiderin deposits in the cytoplasm of macrophages in the red pulp, and sometimes in the white pulp as well; thus, iron pigments are a typical finding (i.e., hemosiderin and ferritin) in the macrophages of the red pulp (Cesta, 2006; Melbius \& Kroal, 2005; Saito et al., 1988).

\section{Splenectomy (SPLY):}

In general, the whole tissue of the spleen is soft and quite fragile, therefore, in the daily life of humans or vertebrate animals often occurs ruptures and damages to the spleen due to traffic accidents, arm shots, fall down from high altitudes, extreme sports, abdominal blows, rib fractures or even for practicing sports with full contact. In the event a person suffers an accident like these, a surgery name SPLY must be soon practiced to that person to take out the damaged spleen, totally or partially, and to stop the process of bleeding (Whitlock, 2016).

Although preservation of the spleen following abdominal trauma and spleen-preserving surgical procedures have received important attention in recent years, about 22,00o SPLYs are conducted annually (for all causes) in the USA (Crary \& Buchanan, 2009) even by laparoscopy or robotic techniques (Olson, 2016). Total spleen removal in trauma patients is becoming less common as a result of more conservative non-operative management of splenic injury (Olson, 2016). The main complications exhibited by patients that suffer a SPLY are- a) bacterial infections by encapsulated microorganisms and Gram-negative bacteria (Gallagher, 2001; CheslynCurtis et al., 1988; Looke \& Runnegar, 2012; Morgan \& Tomich, 2012), b) decreased phagocytic action, c) low IgM production, d) leukocytosis and thrombocytosis in particular at the portal vein (Toutouzas et al., 2002; Khan et al., 2009; Vannucchi \& Barbui, 2007) and e) it appears that SPLY increases the risk of developing cancer in splenectomized patients (Crary \& Buchanan, 2009; Linet et al., 1996; Mellemkjoer et al., 1995).

\section{SPLYand Hemoparasitic Diseases:}

Different hemoparasites present in tropical and subtropical regions, i.e. Plasmodium, Babesia, Anaplasma, Leishmania, Ehrlichia, Trypanosoma, and Borrelia, and other protozoan parasites asHepatozoon, or Neospora, are able to infect susceptible persons, but those infections are mainly controlled either with specific drugs and/or immunologically controlled post infection by specific immune cells, and organs like the spleen, since it acts as a constant blood filter (Rosner et al., 1984; Mark et al., 1997; Vannier et al., 2012). However, any splenectomized person who lives in geographic areas where there are high risks of being infected with hemoparasites of those genera, either after being bitten by infected mosquitos, ticks or fleas, or after blood transfusions, may develop an acute parasitemia, and simultaneously an acute clinical disease which can be fatal if the patient is not treated on time (Vannier et al., 2012; Pasternak, 2016). In addition to specif ic symptoms, asplenic patients or animals may suffer reinfections with the same or with a different hemoparasite, and as a result to show some hemolytic disorders like Disseminated Intravascular Coagulation (DIC), hemolytic anemia, thrombosis or thrombocytopenia (Gupta et al., 2013; Kettner et al., 2003; Vannier et al., 2008; Melendez et al., 2014). In the tropics is common to have peaks of epidemic diseases due to three main factors: 1) the large population of infected hematophagous arthropods developed during the rainy season, which often are hemiparasite-borne vectors infecting people, 2) the poor socio-economical habitat where those people live on, and 3) the lack of systematic control methods for those arthropods in the environment (Melendez, 200o). Consequently, the prevalence of those hemoparasitic diseases in the Neotropical region is higher compared with other ecological regions; nonetheless, as long as people are immunocompetent few cases of the fatal disease are registered, i.e. babesiosis, ehrlichiosis, or anaplasmosis. The spleen in particular in alliance with all the other lymphatic organs, plus the lymphocytic cells, and the MPS act as a defensive barrier against those systemic topical infections; therefore, an additional question might raise up: May the spleen have a role in the control of an arthropod vector of a hemoparasite in the tropics? This question was recently address using the 
experimental model Amblyomma cajennense (Acari: Ixodidae) infecting intact and splenectomized laboratory rats (Rattus norvegicus) (Melendez et al., 2014), with the following results: 1) The total amount of engorged larvae and of collected nymphae of A.cajennense was 3.5 times greater in the experimental group (SPLY rats) than in the control group (Intact ones); 2) The histopathologic diagnosis of spleen samples from A.cajennense infected rats showed a greater amount of large lymphoid follicles with hyperplasia of lymphocytes, plasmocytes, and macrophages, which means activated splenic focuses. On the contrary, a similar tissue reaction was not observed in spleen samples of A.cajennense uninfected rats; 3) This histopathologic diagnosis found in the spleen of infected rats seems to point out that the spleen appears to have also an active role in the control of the population of A.cajennense evolutive stages, at least in Sprague-Dawley rats; and 4) An hematologic study performed to rats at both groups showed a marked thrombocytopenia in all A.cajennense infected rats, which appears to be caused either by pharmacological factors inoculated into the host with the saliva during the bloodsucking process and/or due to the SPLY. Overall, it appears that the splenic tissue and its cells are able to check down even the reproductive rate of anhematophagousecto parasite as the tick A.cajennense, at least in rats. A possible explanation for this immune protective reaction in rats is that the tick saliva carries a complex group of pharmacologically active substances (PHAS), i.e. anticoagulants, antihemostatics, antinflamatories, antivasoconstriction, and aggregation platelet factor (APF), which are inoculated in the rodent bloodstream while ticks are sucking safely bloodmeals (Bowman et al., 1997; Bowman \& Sauer, 2004); however, most of these PHAS would act as foreign antigens which are recognized by the rat's immune system mounting a humoral and cellular immune response against those antigens secreted by an ectoparasite like $A$. cajennense.

\section{Conclusions:}

Since past years the spleen was considered as a secondary lymphatic organ, which could be surgically removed from the abdominal cavity of traumatized patients with low or none risks for their lives. This medical view has changed in the last 20 years as long as more scientif ic advances occur about the immunological, hematological, and protective functions carried out by the spleen as an essential organ included anatomically in the circulatory system. Nowadays most SPLYs are partially practiced unless a previous medical diagnosis decides that the extraction must be complete to preserve the patient's life. Long last clinical studies has shown that splenectomized patients not only are highly prone to be infected with bacteria like Streptococcus pneumonia, with other pathogenic viruses, and dangerous tropical hemoparasites, but also that they often suffer hemolytic disorders, thrombocytosis, immune hemolytic anemia, immune thrombocytopenia (Gupta et al., 2013; Cox et al., 2012), and even cancer, which are life-threatening conditions if appropriate treatments are not soon issued (Kristinsson et al., 2013; Hull et al., 1988).

\section{References:}

Abbas, A.K., Lichtman, A.H.H., Pillai, S. (2015): Cellular and Molecular Immunology. 8th edition. Pub. by: Elsevier Editorial. NY. London. p.13-34.

Bowman, A.S., Coons, L.B., Nedham, G.R., Sauer, J.R. (1997): Tick Saliva: recent advances and implications for vector competence. Med. Vet. Entomol., 11 (3): 277-85.

Bowman, A.S., Sauer, J.R. (2004): Tick salivary glands: function, physiologyand future. Parasitology, 129 (S):567-81.

Cesta, M.F. (2006): Normal structure, functions, and histology of the spleen. Toxicol. Pathol.,34(5):455-465.

Cheslyn-Curtis, S., Aldrige, M.C., Biglin, J.E., Dudley, H.A.F. (1988): Effect of splenectomy on Gram-negative bacterial clearance in the presence and abscense of sepsis. Br. J. Surg. 75(2): 177-80.

Cox, J., Mota, J., Sukupolvi-Petty, S., Diamond, M.S. \& Rico-Hesse, R. (2012): Mosquito bite delivery of dengue virus enhances immunogenicity and pathogenesis in humanized mice. I. Virol.,86 (14): 7637-7649.

Crary, S.E. \& Buchanan, G.R. (2009): Vascular complications after splenectomy for hematologic disorders. Blood, 114:2861-2868.

Delves, P., Martin, S., Burton, D. \& Roit, I. (2014): Inmunología Fundamentos. Pub. by: Editorial Médica Panamericana, pp 548.

Gallagher, F. (2001): Streptococus infection and splenectomy. Lancet, 357:(9262).

Golub, R. \& Cumano, A. (2013): Embryonic hematopoiesis. Blood Cells Mol. Diseas., 51 (4):226-31.

Gupta, N.K., Bansal, S.B., Jain, U.C. \& Sahare, K. (2013): Study of thrombocytopenia in patients of malaria. Trop. Parasitol., 3(1): 58-61.

Hull, C.C., Galloway, P., Gordon, N., Gerson, S.L., Hawkins, N., Stellato, T.A. (1988): Splenectomy and the induction of murine colon cancer. Arch. Surg., 123(4):4624.

Kettner, F., Revers, F. \& Miller, D. (2003): Thrombocytopenia in canine babesiosis and its clinical usefulness. J. S. Afr. Vet. Assoc. 74(3):63-68.

Khan, P.N., Nair, R.J., Olivares, J., Tingle, L.E., Li, Z. (2009): Postsplenectomy reactive thrombocytosis. Proc (Bayl Univ. Med Cent.). 22(1): 9-12.

Kristinsson, S.Y., Gridley, G., Hoover, R.N., Check, D. \& Landgren, O. (2014): Long-term risks after splenectomy among 8,149 cancer-free American veterans: a cohort study with up to 27 years follow-up. Haematolo., 99:392-398.

Linet, M.S., Nyren, O., Gridley, G., Mellemkjoer, L., Mclaughlin, J.K., Olsen, J.H., Adam, H.O. \& Fraumeni, J.F. Jr. (1996): Risk of cancer followingsplenectomy. Int.I. Cancer. 66(5):611-616.

Looke, D.F.M. \& Runnegar, N.J. (2012): Splenectomy and sepsis. Med.J.Aust., 196(9):587.

Mark, A. ,Malesker, D.B., Thomas, A., Ruma, P.J., Vuchetich, P., Murphy, J.S. \& Alegent, P.W. Rhodesian trypanosomiasis in a splenectomized patient. Am. J. Trop. Med. Hyg., 61(3):428430. 
Melbius, R.E. \& Kroal, G. (2005): Structure and function of the spleen. Nat. Rev. Immunol., 5: 606-616.

Meléndez, R.D., Meléndez, A.C., Marín, S., Torres, A., Fortis, M., Granda, F. \& Mujica, F. (2014): Fases de larva y ninfa de Amblyommacajennense (Acari: Ixodidae) en ratas (Rattusnorvegicus) esplenectomizadas e intactas: estudio de casos. Rev. Cientif. FCV-LUZ., XXIV(5): 421-427.

Meléndez, R.D. (200): Future perspectives on veterinary hemoparasite research in the tropics at the start of this century.Ann. N. . . . Acad. Sci., 916:253-258.

Mellemkjoer, L., Olsen, J.H., Linet, M.S., Gridley, G. \& Mclaughlin, J.K. (1995): Cancer risk after splenectomy. Cancer. 75(2): 577-583.

Molina, D.K. \& DiMaio, V.J. (2012): Normal organ weights in men: Part II- the brain, lungs, liver, spleen, kidneys- $\underline{A m . J . ~ F o r e n s i c . ~}$ Med. Pathol., 33(4):368-372.

Moore, K.L. (2013): Anatomía con Orientación Clínica. $7^{\underline{a}}$ ) edición. Lippincott, Williams \&Wilkins (LWW). Philadelphia, PA, USA.pp. 1327.

Morgan, T.L. \& Tomich, E.B. (2012): Overwhelming postsplenectomy infection (OPSI).J. Emerg. Med., 43(4): 758-763.

Netter, F.H. (2011): Atlas de Anatomía Humana. 5a) edición. Editorial Elsevier, Amsterdam, Londres.pp. 624.

Olson, Ch. (2016): Robotic splenectomy. Spleen removal. YouTube. (Scripps San Diego. 6:53 min. Visited:11/16/2016).

Patterson, K.D., Drysdale, T.A. \& Krieg, P.A. (200o): Embryonic origins of spleen asymmetry. Development., 127(1):167-175.

Rosner, F., Zarrabi, M.H., Benach, J.L. \& Habicht, G.S. (1984): Babesiosis in splenectomized adults. Review of 22 reported cases. Am.L.Med., 76(4):696-701.
Saito, H., Yokoi, Y., Watanabe, S., Tayima, J., Kroda, H. \& Namihisa, T. (1988): Reticular meshwork of the spleen in rats studied by electron microscopy. Am.J.Ant., 181: 235-252.

Satodate, R., Tanaka, H., Sasou, S., Sakuma, T. \& Kaizuka, H. (1986): Scanning electron microscopical studies of the arterial terminals in the red pulp of the rat spleen. $\underline{\text { Anat. }}$ Rec. 215:214-216.

Swirski, F.K., Nahrendorf, M., Etzrodt, M. Wildgruber, M., Cortez-Retamozo, V., Panizzi, P., Figueiredo, J.L., Kohler, R.H., Chudnovskiy, A., Waterman, P., Aikawa, E., Mempel, T.R., Libby, P., Weissleder, R. \& Pittet, M.J. (2009): Identification of splenic reservoir monocytes and their deployment to inflammatory sites. Science, 325 (5940): 6126.

Toutouzas, K.G., Velmahos, G.C., Kaminski, A., Chan, L. \& Demetriades, D. (2002): Leukocytosis after post traumatic splenectomy: a physiologic evento r sign of sepsis? Arch. Surg., 137(8):924-928.

Vannucchi, A.M. \& Barbui, T. (2007): Thrombocytosis and thrombosis. Am. Soc. Hematol. Educ. Progam., 2007: 363370.

Vannier, E., Gewurz, B.F., Krause, P.J. (2008): Human babesiosis. Infect. Dis. Clin. NorthAm., 22(3):469-470.

Vannier, E. \& Krause, P.J. (2012): Human Babesiosis. N. Engl. J. Med., 366:2397-2407.

Whitlock, J. (2016): Splenectomy: spleen removal surgery (Online article). https://www.verywell.com/when-is-it-safe-tobathe-after-surgery-3156846 (Visited:11/21/2016).

Zhao, X., Wu, N. \& Hung, L. (2010): Endothelial progenitor cells and spleen: new insights in regeneration medicine. Cytotherapy, 12:7-16. 